\title{
30 Years Aktuelle Dermatologie
}

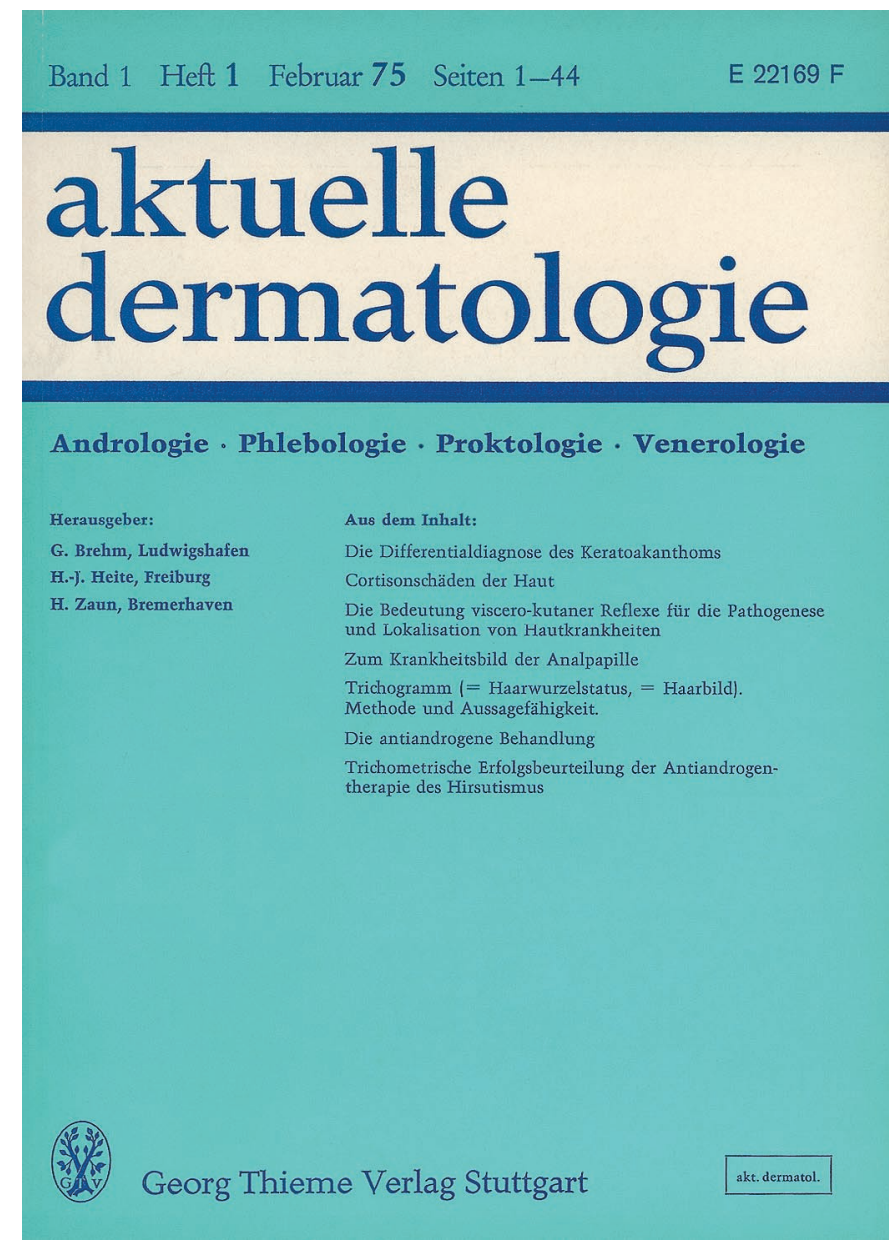

Abb. 1 Die Umschlagseite des ersten Heftes von 1975.
1972 wurde auf Veranlassung von Dr. med. h.c. Günther Hauff die Aktuelle Dermatologie gegründet. Mit der redaktionellen Arbeit wurde G. Brehm beauftragt. Weitere Gründungsmitglieder waren H. J. Heite und H. Zaun.

Zur damaligen Zeit bestand bereits eine Reihe von renomierten dermatologischen Zeitschriften mit festem Leserkreis und insgesamt hohen Auflagen. Das Risiko einer Neugründung war groß. So bestanden auch gewisse Widerstände gegen die neue Zeitschrift mit der Begründung, es gebe schon genügend dermatologische Blätter und das Angebot an druckfähigen Beiträgen sei insgesamt zu gering.

Wir begannen bescheiden mit einer kleinformatigen Ausgabe (DIN A5), brachten aber schon früh durchgehend farbige Abbildungen, die für den Autor kostenlos waren (Abb.1). Vom 7. Jahrgang an wurde dann auf das übliche internationale Magazinformat umgestellt. Die Sprache war und ist Deutsch, natürlich mit einem englischen Abstract, da die Aktuelle Dermatologie sich an deutschsprachige Leser richtet.

Die Aktuelle Dermatologie erweiterte die Reihe der Fortbildungszeitschriften des Georg Thieme Verlags. Als Arbeitsziel erklärten die Herausgeber, „es soll ausschließlich Anliegen der Aktuellen Dermatologie sein, über die neuesten Forschungsergebnisse zu unterrichten, die für die tägliche praktische Arbeit bedeutsam sind, und das diagnostische und therapeutische Wissen zu vertiefen." Somit werden als Zielgruppen sowohl klinisch tätige als auch niedergelassene Dermato-Venerologen angesprochen. 
Beiträge aus dem gesamten Fachgebiet einschließlich Andrologie, Allergologie, Berufsdermatosen, Dermatoonkologie, Infektiologie, Mykologie, Phlebologie, Photodermatologie und Proktologie finden sich in der Aktuellen Dermatologie. So ist sie auch Organ der Arbeitsgemeinschaft Dermatologische Onkologie und Organ der Deutschen Gesellschaft für Photobiologie.

1999 kam es zur Vereinigung mit der Zeitschrift für Dermatologie und damit zur wertvollen Mitarbeit der Herausgeber dieser Zeitschrift.

Die Herausgeber und Mitarbeiter der Aktuellen Dermatologie waren stets bemüht, in ihrer Arbeit auf dem Laufenden der neuesten Forschungsergebnisse zu bleiben und sowohl wissenschaftlich als auch praktisch ausgerichtete Beiträge zu liefern. Übersichten, Originalarbeiten, Kasuistiken, Editorials, Referate aus anderen Fachzeitschriften, Buchbesprechungen, Interviews und Sonderkapitel wie Kurz und Lehrreich, Der interessante Fall, Eine Klinik im Blickfeld, die Knacknuss u. a. zeigen zusammengefasst die Vielfalt des Inhalts auf. Die Autoren der Beiträge kamen aus fast allen Hautkliniken des deutschsprachigen Raums, dazu kamen erfreulicherweise auch Arbeiten niedergelassener Dermatologen und anderer Disziplinen.
Im Laufe der Jahre haben eine Reihe von weiteren Kollegen im Herausgeberteam mitgearbeitet so T. Bieber, E. B. Bröcker, P. Elsner, S. Goerdt, M. Goos, M. Herbst, E. G. Jung, H. Meffert, I. Moll und R. Stadler. (s. Tab. 1 auf S. 239.) Bleibt noch zu ergänzen, dass die Redaktion im Jahre 2002 von G. Brehm [1] auf R. Stadler und ab 2004 auf E. G. Jung überging.

30 Jahre sind verglichen mit dem Bestehen der alten, klassischen dermatologischen Zeitschriften, die z.T. noch aus dem 19. Jahrhundert stammen, eine kurze Zeitspanne. Dennoch sind die Herausgeber stolz darauf, dass es ihnen gelungen ist allen Widerständen zum Trotz über die Jahre hinaus offenbar gute Arbeit geleistet zu haben [2]. Die immer wieder geäußerte Beliebtheit der Aktuellen Dermatologie soll Ansporn und Verpflichtung sein, die Arbeit wie bisher mit Unterstützung des Georg Thieme Verlags fortzusetzen.

\section{Literatur}

${ }^{1}$ Jung EG, Hauff A. Prof. Georg Brehm tritt nach 27 Jahren als Herausgeber unserer Fachzeitschrift „Aktuelle Dermatologie“ zurück. Akt Dermatol 2001; 27: 383-384

2 Zaun HO. 25 Jahre „Aktuelle Dermatologie“ Akt Dermatol 1999; 25: $363-364$ 